\title{
Stimuli-disassembling gold nanoclusters for diagnosis of early stage oral cancer by optical coherence tomography
}

Chang Soo Kim ${ }^{1,2}$, Dominique Ingato ${ }^{1}$, Petra Wilder-Smith ${ }^{2}$, Zhongping Chen ${ }^{1,2,3,6^{*}}$ and Young Jik Kwon ${ }^{1,3,4,5,7^{*}}$ (1)

\begin{abstract}
A key design consideration in developing contrast agents is obtaining distinct, multiple signal changes in diseased tissue. Plasmonic gold nanoparticles (Au NPs) have been developed as contrast agents due to their strong surface plasmon resonance (SPR). This study aims to demonstrate that stimuli-responsive plasmonic Au nanoclusters (Au NCs) can be used as a contrast agent for optical coherence tomography (OCT) in detecting early-stage cancer. Au NPs were clustered via acid-cleavable linkers to synthesize Au NCs that disassemble under mildly acidic conditions into individual Au NPs, simultaneously diminishing SPR effect (quantified by scattering intensity) and increasing Brownian motion (quantified by Doppler variance). The acid-triggered morphological and accompanying optico-physical property changes of the acid-disassembling Au NCs were confirmed by TEM, DLS, UVNis, and OCT. Stimuli-responsive Au NCs were applied in a hamster check pouch model carrying early-stage squamous carcinoma tissue. The tissue was visualized by OCT imaging, which showed reduced scattering intensity and increased Doppler variance in the dysplastic tissue. This study demonstrates the promise of diagnosing early-stage cancer using molecularly programmable, inorganic nanomaterial-based contrast agents that are capable of generating multiple, stimuli-triggered diagnostic signals in early-stage cancer.
\end{abstract}

Keywords: Optical contrast agent, Gold nanoparticles, Acid-transforming nanoclusters, Optical coherence tomography, Oral cancer

\section{Introduction}

Unique and tunable optical properties [e.g., surface plasmon resonance (SPR) effect] without obstruction by photo-bleaching or photo-blinking have made plasmonic inorganic nanoparticles (NPs) an attractive, popularlyinvestigated nanomaterial for biomedical imaging applications including optical imaging [1]. Resonant excitation of plasmonic inorganic NPs leads to a large enhancement of the incident electromagnetic field at the NP surface for nonlinear optical spectroscopies such as surface enhanced Raman spectroscopy. [1,2] One category of

\footnotetext{
*Correspondence: z2chen@uci.edu; kwonyj@uci.edu

${ }^{6}$ Department of Chemical Engineering and Materials Science, University of California, Irvine, 1002 Health Sciences Rd, Irvine, CA 92617, USA

${ }^{7}$ Department of Pharmaceutical Sciences, University of California, Irvine, 132 Sprague Hall, Irvine, CA 92697, USA

Full list of author information is available at the end of the article
}

plasmonic NPs, gold ( $\mathrm{Au}$ ) NPs do not induce cytokine secretion, making them highly biocompatible and applicable to a number of delivery, sensing, and imaging applications [3-6]. Au NPs have been most intensively investigated for imaging applications over the last few decades; well-established methods of Au NP synthesis and surface modifications have allowed for control over their morphology-dependent optical properties [7-13]. Among many imaging applications, optical coherence tomography (OCT) is a fast, non-invasive, and high resolution imaging modality that uses a Michelson's interferometer with a low coherence light source [14]. OCT's two- and three-dimensional high resolution imaging capability is well-suited for detecting specific stages of diseases such as cancer [15-18]. However, the challenge of obtaining disease-specific molecular contrast undermines the many promising features of OCT [19-22]. This 
limitation particularly affects OCT diagnostic performance for early-stage cancer; however, it can potentially be overcome by using optical contrast agents such as $\mathrm{Au}$ NPs [3, 23-26]. For example, significantly enhanced OCT contrast in vivo was demonstrated by administering antiepidermal growth factor receptor-conjugated Au NPs via a multimodal delivery method to neoplastic tissues [27].

Plasmonic Au nanoclusters (NCs) that transform their physical and optical properties upon detecting a stimulus (e.g., mildly acidic $\mathrm{pH}$ of tumor tissue [28-30]) in a diseased area are highly promising OCT contrast agents due to their ability to improve OCT imaging with enhanced SPR effects [31, 32]. Moreover, $\mathrm{pH}$-transforming systems of Au NPs have previously been described in the literature; one approach includes Au NPs composited in $\mathrm{pH}$ responsive polymers that expand under acidic conditions due to changes in electrostatic forces $[33,34]$. In this study, we synthesized Au NCs by using an acid-cleavable linker to cluster individual Au NPs.

We hypothesized that the mildly-acidic tumor microenvironment would disassemble acid-degradable Au NCs to individual $\mathrm{Au}$ NPs, resulting in OCT's Doppler variance frequency shift due to increased Brownian motion as well as decreased intensity signals upon decreasing SPR effect. Acid-degradable Au NPs were clustered via acid-cleavable linkers to pinpoint mildly acidic tumor tissue $[28,30]$. Spectral domain (SD) and Doppler variance (Dv) OCT modes were used to characterize morphological changes from clusters (scattering-dominant with SPR effect; slow Brownian motion) to dispersed $\mathrm{Au}$ NPs (absorption-dominant with SPR effect; fast Brownian motion) (Fig. 1). The Au NCs generate high scattering signal in normal tissue due to strong inter-particle SPR effect while substantially reducing scattering signal in mildly acidic tumor tissue upon disassembly to small individual Au NPs. Simultaneously, the disassembled

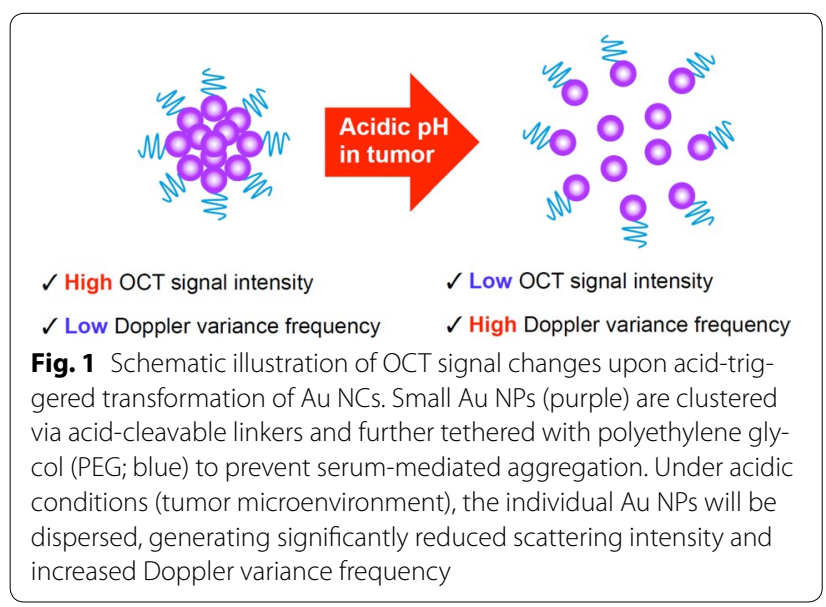

$\mathrm{Au}$ NPs in tumor tissue increases the Doppler variance frequency spectrum due to their small size (increased Brownian motion), which can be quantified by DvOCT. [35] Therefore, the stimuli-triggered disassembly of $\mathrm{Au}$ NCs can be used to visualize a neoplastic site with high resolution by multiple optical signal changes: the loss of scattering signal and an increase in Doppler variance frequency spectrum $[36,40]$. In vivo OCT imaging pinpointed the site of early-stage oral dysplasia via acid-disassembly of $\mathrm{Au} \mathrm{NCs}$ and validated the hypothesis of this study.

\section{Methods/experimental}

\subsection{Materials}

Hydrogen tetrachloroaurate (III) trihydrate $\left(\mathrm{HAuCl}_{4} \cdot 3 \mathrm{H}_{2} \mathrm{O}\right)$ and trisodium citrate dihydrate $\left(\mathrm{Na}_{3} \mathrm{C}_{6} \mathrm{H}_{5} \mathrm{O}_{7} \cdot 2 \mathrm{H}_{2} \mathrm{O}\right)$ were purchased from Sigma Aldrich (Milwaukee, WI). N-Succinimidyl 3-(2-pyridyldithio)propionate (SPDP) and amino PEG monomethyl ether $(2 \mathrm{kDa})$ were purchased from Pierce (Rockford, IL). 2-Aminoethanol, pyridinium $p$-toluenesulfonate, ethyl trifluoroacetate, and 2-methoxypropene were supplied by Acros (Morris Plains, NJ). Dimethly sulfoxide (DMSO), sodium azide, and bovine serum albumin were purchased from Fisher Scientific (Fair Lawn, NJ). Dithiothreitol (DTT) was supplied by Fisher Scientific (Pittsburgh, PA) and diethylene glycol bis (3-amino propyl) ether was obtained from TCI (Tokyo, Japan). Carboxy-SNARF-1 (SNARF) was purchased from Invitrogen AB (Frolunda, Sweden). A carbon-coated copper TEM grid was purchased from Electron Microscopy Sciences (Hatfield, PA).

\subsection{Synthesis of Au NPs}

Citrate-capped Au NPs (15 nm in diameter) were synthesized by following a previously reported protocol with slight modifications [37]. Briefly, 100 milliliters of $1 \%(\mathrm{w} / \mathrm{w}) \mathrm{HAuCl}_{4} \cdot 3 \mathrm{H}_{2} \mathrm{O}$ in Milli-Q water $(18.2 \mathrm{M} \Omega$ ) was heated to its boiling point $\left(100{ }^{\circ} \mathrm{C}\right)$ and added dropwise with $4 \mathrm{~mL}$ of $1 \%(\mathrm{w} / \mathrm{v}) \mathrm{Na}_{3} \mathrm{C}_{6} \mathrm{H}_{5} \mathrm{O}_{7} \cdot 2 \mathrm{H}_{2} \mathrm{O}$ in Milli-Q water (18.2 $\mathrm{M} \Omega$ ) with vigorous stirring. Upon addition of $\mathrm{Na}_{3} \mathrm{C}_{6} \mathrm{H}_{5} \mathrm{O}_{7} \cdot 2 \mathrm{H}_{2} \mathrm{O}$, the color of the mixture changed to deep red instantaneously. After reflux for an additional $15 \mathrm{~min}$, the resulting solution was cooled down to room temperature with continuous stirring. After overnight incubation at $25{ }^{\circ} \mathrm{C}$, the resulting $\mathrm{Au}$ NPs were characterized by using a Phillips CM 20 transmission electron microscope (TEM) (Philips Electronic Instruments, Mahwah, NJ) and ZEN3600 Zetasizer dynamic light scattering (DLS) particle analyzer (Malvern Instruments, Worcestershire, UK). It was shown that Au NPS were $15 \mathrm{~nm}( \pm 0.3 \mathrm{~nm})$ in diameter and spherical in shape (TEM). The concentration of the resulting Au NPs was 
calculated to be $3.44 \times 10^{12}$ particles $/ \mathrm{mL}$ using a previously described UV/Vis spectroscopic method [38]. All glassware used in the Au NP synthesis was cleansed in aqua regia, 3:1 (v/v) $\mathrm{HCl}: \mathrm{HNO}_{3}$, and thoroughly rinsed with deionized (DI) water.

\subsection{SPDP-activation of Au NPs}

Ten milliliters of Au NPs $\left(3.44 \times 10^{12}\right.$ particles $\left./ \mathrm{mL}\right)$ were washed with Milli-Q water $(18.2 \mathrm{M} \Omega$ ) by repeated centrifugation at $10,000 \mathrm{rpm}$ for $30 \mathrm{~min}$ and then redispersed in Milli-Q water (18.2 M $\Omega$ ). After the 2 times wash steps, Au NPs were re-dispersed in $10 \mathrm{~mL}$ of $0.02 \mathrm{M}$ sodium bicarbonate buffer ( $\mathrm{pH}$ 8.73). Using a peristatic pump, ten milliliters of Au NPs $\left(3.4 \times 10^{12}\right.$ particles/ $\mathrm{mL}$ ) in sodium bicarbonate buffer were steadily added at $7 \mu \mathrm{L} / \mathrm{s}$ to $2 \mu \mathrm{L}$ of $20 \mathrm{mM}$ SPDP solution in dimethyl sulfoxide (DMSO) $\left(4.73 \times 10^{-20} \mathrm{~mol}\right.$ SPDP per Au NP) while the solution was stirred vigorously on ice. The reaction was left stirring at room temperature overnight. Unreacted SPDP was removed by centrifugation twice at 10,000 rpm for $30 \mathrm{~min}$, and SPDP-activated Au NPs were re-dispersed in $10 \mathrm{~mL}$ of $0.02 \mathrm{M}$ sodium bicarbonate buffer and briefly sonicated in a sonication bath for $30 \mathrm{~s}$ [39]. When exposed to dithiothreitol (DTT), SPDP cleaves; this concept was used to quantify SPDP conjugation. SPDP conjugation of Au NPs was quantified by the release of a pyridine-2-thione group when incubated with dithiothreitol (DTT). Briefly, $1.5 \mathrm{~mL}$ of SPDPactivated $\mathrm{Au} \mathrm{NP}$ solution $\left(3.4 \times 10^{12}\right.$ particles $\left./ \mathrm{mL}\right)$ was washed twice with DI water by repeated centrifugation at 10,000 rpm for $30 \mathrm{~min}$ and re-dispersion. Seventy-five microliters of $15 \mathrm{mg} / \mathrm{mL}$ DTT in DI water were added to $0.75 \mathrm{~mL}$ of SPDP-Au NP solution, followed by incubation for $15 \mathrm{~min}$ at room temperature. The released amount of pyridine-2-thione was quantified by the absorbance at $343 \mathrm{~nm}$ using a Varian Cary $50 \mathrm{UV} /$ Vis spectrophotometer (Varian Inc., Palo Alto, CA). After subtracting the background absorbance of a DTT-free SPDP-Au NP solution at the same concentration, SPDP-conjugation was calculated to be $5.34 \times 10^{-22} \mathrm{~mol}(=321 \mathrm{~mol})$ of SPDP per Au NP. The resulting SPDP-activated Au NPs were kept at $4{ }^{\circ} \mathrm{C}$ without exposure to light.

\subsection{Au NC synthesis and characterization}

Seven hundred fifty microliters of SPDP-activated $\mathrm{Au}$ NPs $\left(6.8 \times 10^{12}\right.$ particles $\left./ \mathrm{mL}\right)$ were added slowly into acid-cleavable diaminoketal (DAK) solution in $0.02 \mathrm{M}$ sodium bicarbonate solution on ice with strong stirring. Then, the mixture remained at room temperature overnight with continuous stirring. The unreacted cross-linkers were washed away by centrifugation at $8000 \mathrm{rpm}$ for $10 \mathrm{~min}$ and re-dispersed in $0.02 \mathrm{M}$ sodium bicarbonate solution. DAK conjugated Au NPs were characterized by dynamic light scattering (DLS) and UV/vis spectrometer. $100 \mu \mathrm{L}$ of DAK conjugated Au NPs were slowly added to $10 \mathrm{~mL}$ of SPDP-activated Au NPs on ice, and the mixture was kept stirring overnight. The final $\mathrm{Au}$ clusters were added to $160 \mu \mathrm{L}$ of amine-functionalized PEG ( $M W=2 \mathrm{k} \mathrm{Da}, 10 \mathrm{mg} / \mathrm{mL}$ ) with strong stirring. The acid degradable $\mathrm{Au}$ clusters were separated from free, SPDP-activated Au NPs by centrifugation at $6000 \mathrm{rpm}$ for $30 \mathrm{~min}$ [35]. The non-acid degradable (control) $\mathrm{Au}$ clusters were synthesized by the same method except diethylene glycol bis (3-amino propyl) ether $(0.05 \mathrm{~g} / \mathrm{mL})$ was used instead of DAK. The sizes of the Au NPs and $\mathrm{Au}$ NCs were determined using a Phillips CM 20 transmission electron microscope (TEM) (Philips Electronic Instruments, Mahwah, NJ) and ZEN3600 Zetasizer dynamic light scattering (DLS) particle analyzer (Malvern Instruments, Worcestershire, UK). The absorbance of Au NCs was measured using a Varian Cary 50 UV/Vis Spectrophotometer (Varian Inc., Palo Alto, CA).

\subsection{OCT/DvOCT imaging configuration}

Spectral-domain optical coherence tomography (SDOCT) [27] was used to image the scattering and Doppler variance of the acid-degradable and non-acid degradable Au NCs in droplets and hamster cheek pouch tissues. Doppler variance OCT images (DvOCT) were also obtained based on the power spectrum of the temporal fluctuations of the OCT magnitude using the SD-OCT system. Low-coherence light with a $1310 \mathrm{~nm}$ center wavelength and $90 \mathrm{~nm}$ full width at half maximum (FWHM) was used, and imaging depth and depth resolution were $3.4 \mathrm{~mm}$ and $8 \mu \mathrm{m}$ in air, respectively. A 2 -axis scanner with two galvanometers was located at the same sample arm. All SD-OCT and DvOCT images were obtained with the same focal point. A $130 \mathrm{~nm}$ wide spectrum was sampled by a $1 \times 10^{24}$ InGaAs detector array at a $7.7 \mathrm{kHz}$ frame rate. Acid degradable Au-NCs $\left(1.62 \times 10^{9}\right.$ particles $\left./ \mathrm{mL}\right)$ were mixed with $\mathrm{pH} 5$ solution in $37^{\circ} \mathrm{C}$. Then, SD-OCT and DvOCT images were obtained every 30 min with $3 \mu \mathrm{L}$ of aliquot solution for $2 \mathrm{~h}$.

\subsection{OCT/DvOCT imaging of Au NCs incubated in DI water and $\mathrm{pH} 5.0$ acetate buffer}

The acid-degradable and control Au NCs $(960 \mu \mathrm{L}$, $7.05 \times 10^{9}$ particles $/ \mathrm{mL}$ ) were concentrated to $10 \mu \mathrm{L}$ of solution by centrifugation at $2000 \mathrm{~g}$ for $30 \mathrm{~min}$. The resulting solution $(3 \mu \mathrm{L})$ was mixed with $6 \mu \mathrm{L}$ of DI water as a control, and $7 \mu \mathrm{L}$ of the solution was mixed with 14 $\mu \mathrm{L}$ of $\mathrm{pH} 5.0$ acetate buffer to hydrolyze the Au clusters. After $2 \mathrm{~h}$ of incubation at $37^{\circ} \mathrm{C}$, a $3 \mu \mathrm{L}$ aliquot of each sample was dropped on the polyethylene substrate, and OCT/DvOCT images were obtained. The droplet OCT/ 
DvOCT images with different $\mathrm{pH}$ conditions were quantified with the Scion Image Process (Scion Corporation).

\subsection{SNARF-conjugation on Au clusters}

$\mathrm{Au}$ NP clusters were rinsed with Milli-Q water (18.2 M $\Omega$ ) by repeated centrifugation at $6000 \mathrm{rpm}$ for $30 \mathrm{~min}$ and re-dispersion in Milli- $\mathrm{Q}$ water $(18.2 \mathrm{M} \Omega$ ). In order to functionalize with amino groups, $1.5 \mathrm{~mL}$ Au NP clusters $\left(7.05 \times 10^{9}\right.$ particles $\left./ \mathrm{mL}\right)$ in Milli-Q water $(18.2 \mathrm{M} \Omega)$ were then reacted on ice with vigorous stirring with $3.75 \mu \mathrm{L}$ of $0.05 \mathrm{~g} / \mathrm{mL}$ diethylene glycol bis (3-amino propyl) ether in Milli-Q water (18.2 M $\Omega$ ) (molecular ratio of diethylene glycol bis (3-amino propyl) ether to SPDP is $\left.1.4 \times 10^{5}\right)$. After removing unreacted diethylene glycol bis (3-amino propyl) ether by two centrifugation steps $(6000 \mathrm{rpm}$ for $30 \mathrm{~min}), 1.5 \mathrm{~mL}$ of Au NP clusters $\left(7.1 \times 10^{9}\right.$ particles $\left./ \mathrm{mL}\right)$ re-dispersed in Milli-Q water (18.2 M $\Omega$ ) were added to $0.05 \mathrm{~g} / \mathrm{mL}$ SNARF succinimidyl ester in Milli-Q water (18.2 M $\Omega$ ) on ice, followed by stirring at room temperature overnight without exposure to light. SNARF-conjugated Au NP clusters were obtained in Milli-Q water $(18.2 \mathrm{M} \Omega$ ) after removing un-conjugated dyes by centrifugation at $6000 \mathrm{rpm}$ for $30 \mathrm{~min}$. DLS, UV/vis spectrometer, and TEM were used for the characterization after the SNARF-conjugation.

\subsection{In vivo $O C T$ imaging}

To establish the oral cancer model, golden Syrian hamsters (Mesocricetus auratus, Harlan Sprague-Dawley, San Diego, CA) were topically treated with $0.5 \%(\mathrm{w} / \mathrm{v})$ 9, 10-dimethyl-1,2-benzanthracene (DMBA, Sigma) in mineral oil three times per week for 4-6 weeks to induce dysplasia in one cheek pouch in each animal. The contralateral cheek pouch of the hamster received mineral oil application only. All animal protocols were approved by the Institutional Animal Care and Use Committee (IACUC, 97-1972) at the University of California, Irvine. Acid degradable and control Au clusters were synthesized, characterized, and applied on the hamster cheek pouches using a multi-modal delivery method (microneedles for penetration and ultrasound for distribution of Au NCs). OCT/DvOCT was used to observe the optical property changes of stimuli-responsive $\mathrm{Au}$ clusters. All the OCT/DvOCT Imaging was performed in the cheek pouch of the anesthetized hamster by gently clamping the tissues to a microscope stage using a custom-built, ring-shaped clamp. CR3 roller microneedles (MTS dermaroller with $200 \mu \mathrm{m}$ depth; Clinical Resolution Laboratory, Inc., Beverly Hills, CA) were rolled on both the DMBA-untreated and control sides of the hamster cheek pouches three times at three different angles. Then, $200 \mu \mathrm{L}$ of Au cluster solution $\left(7.05 \times 10^{9}\right.$ particles/ $\mathrm{mL}$ ) were administration by dropping it directly into the 1-cm-diameter aperture of the ring-shaped clamp. After 10 min Au cluster topical administration, ultrasonic force $\left(0.3 \mathrm{~W} / \mathrm{cm}^{2}\right.$ of $\left.1 \mathrm{MHz}\right)$ was applied to the cheek pouch using the Dynatron 125 ultrasonicator (Dynatronics Corporation, Salt Lake City, UT) for $1 \mathrm{~min}$ in the presence of ultrasound gel, followed by OCT/DvOCT imaging. After OCT/DvOCT imaging, all the OCT/DvOCT images were quantified with the scion image process (Scion Corporation).

\section{Results and discussion}

\subsection{Synthesis and characterization of acid-disassembling} Au NCs

In order to meet the design goals of this study, Au NCs needed to be composed of small Au NPs of the minimally required size for detection by OCT and DvOCT. Although higher OCT/DvOCT signals can be obtained using large Au NPs [41], the resulting Au NCs should be small enough (e.g., less than $100 \mathrm{~nm}$ [42]) to penetrate and readily disperse in target tissues. In a previous study, we demonstrated that $\sim 70 \mathrm{~nm} \mathrm{Au} \mathrm{NPs} \mathrm{could} \mathrm{be}$ detected at the site of early-stage oral dysplasia in vivo after topical delivery by combined microneedle (penetration through stratum cornea) and ultrasound (dispersion in oral dysplasia) delivery [27]. Therefore, the design goal of this study was to create Au NCs that were smaller than $100 \mathrm{~nm}$ and able to disassemble into individual $15 \mathrm{~nm} \mathrm{Au}$ NPs under mildly acidic conditions.

Briefly, $15 \mathrm{~nm}$ Au NPs were synthesized using the same method that was previously reported with slight modifications [27]. Then, the thiol groups on the NP surface were conjugated with $\mathrm{N}$-succinimidyl 3-(2-pyridyldithio)propionate (SPDP), a heterofunctional cross-linker with amine-reactive NHS and thiol-reactive pyridyldithiol groups, generating Au NPs with NHS groups on their surface (Au NP-SPDP). Addition of excess acid-cleavable ketal cross-linker (diaminoketal [DAK]) to the Au NPSPDP, followed by removal of unreacted DAK, resulted in $\mathrm{Au}$ NPs with amine groups conjugated on the surface (Au NP-DAK). The DAK cross-linker was synthesized as previously reported [43]. The amine groups on the surface of Au NP-DAK were then used to anchor Au NP-SPDP via their surface NHS groups, resulting in Au NP-DAKs clustered by Au NP-SPDP (Au NP-DAK-Au NP-SPDP). Finally, the remaining NHS groups on the surface of the surrounding Au NPs were further conjugated with polyethylene glycol (PEG) with an amine group. PEG was conjugated on the surface of $\mathrm{Au} \mathrm{NCs}$ to prevent aggregation, particularly under biological/physiological conditions [44]. The synthesis of acid-disassembling Au NCs is illustrated in Fig. 2. Non-acid-degradable (control) Au NCs were also synthesized by the same method except diamino cross-linker without a ketal linkage. More 


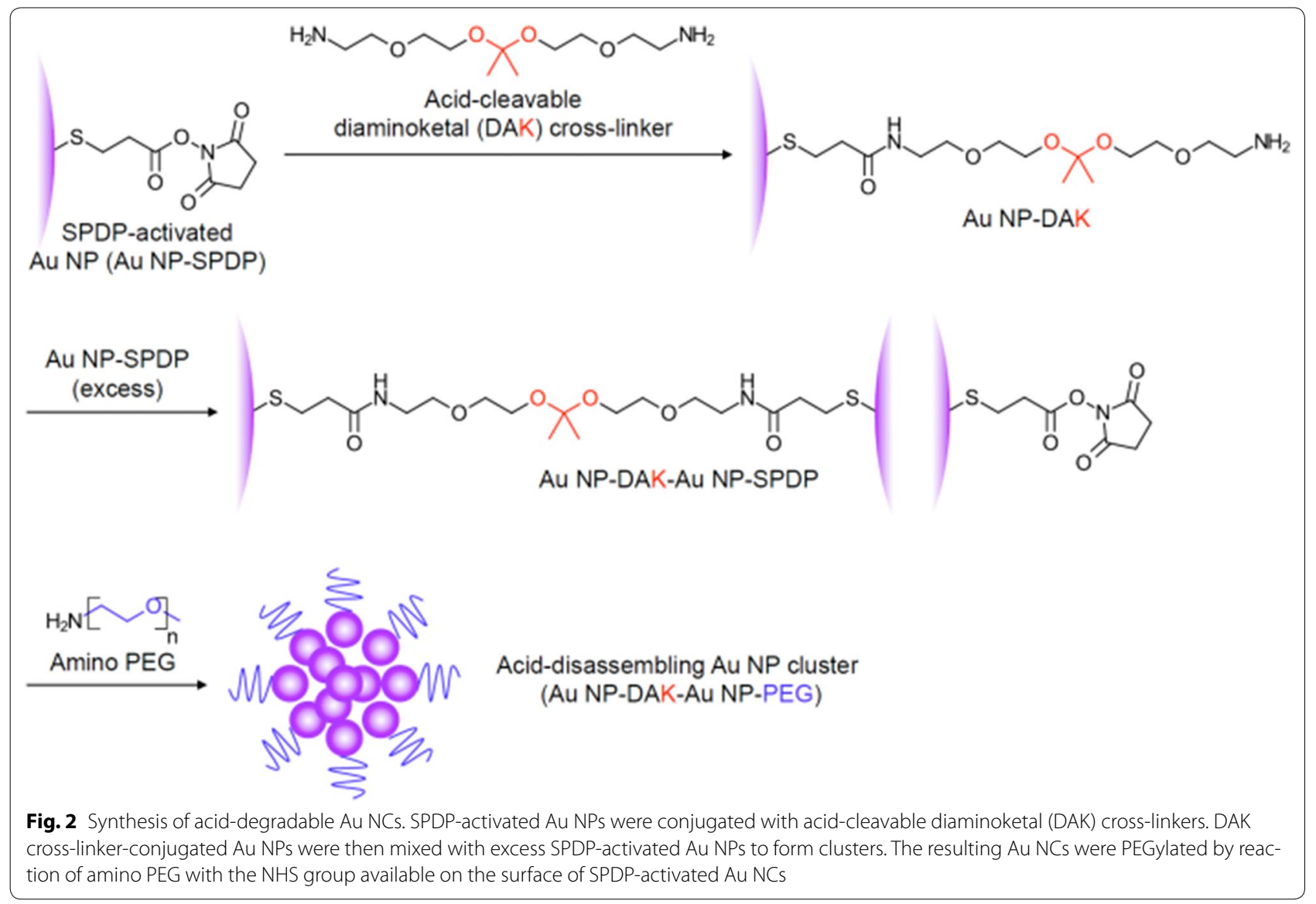

specifically, diethylene glycol bis (3-amino propyl) ether $(0.05 \mathrm{~g} / \mathrm{mL})$ were used instead of DAK. Au NP size, the ratio of Au NP-SPDP to Au NP-DAK, and the molecular weight of PEG control the size of the resulting Au NCs.

\subsection{Acid-responsive transformation of Au NCs and optical signal changes}

As intended, the resulting acid-disassembling Au NCs were approximately $85 \mathrm{~nm}$ in diameter, while non-aciddisassembling Au NCs were $80 \mathrm{~nm}$. The sizes of particles were determined by dynamic light scattering (DLS), followed by transmission electron microscopy (TEM). In this proof-of-concept experiment, acid-responsive disassembly of the resulting Au NCs was investigated by incubating them in DI water and an acetate buffer $(100 \mathrm{mM}$; $\mathrm{pH}$ 5.0), a conventionally used acidic condition. As shown in TEM images (Fig. 3a, b), acid-responsive Au NCs rapidly disassembled at $\mathrm{pH}$ 5.0. The acid-disassembly of Au NCs from covalently clustered Au NPs in DI water (Fig. 3a) to individual Au NPs with slight aggregation at $\mathrm{pH} 5.0$ (Fig. 3b) was also indicated by appearance of split size peaks in DLS measurement (91\% at $64 \mathrm{~nm}$ and $9 \%$ at $6 \mathrm{~nm}$ in intensity) (Fig. 3c). Simultaneously, the SPR absorption peak was shifted from 531 to $527 \mathrm{~nm}$ (Fig. 3d). As shown in Fig. 3b, there were only slightly aggregated Au NPs (40-80 nm) and individual Au NPs $(15 \mathrm{~nm})$. Therefore, the peak for small particles $(6 \mathrm{~nm})$ (Fig. 3c) could be an artifact in DLS measurement. DLS determines particle sizes based on lumped Brownian motion, rather than tuning for individual sizes. Therefore, signal intensity generated by larger particles is greatly weighted over those generated by smaller ones. Taken together, the results shown in Fig. 3 confirmed the acidtriggered morphological changes of Au NCs. pH 5.0 was used in this study as a commonly used acidic condition in many reports. The disassembling kinetics of Au NCs would be affected by different $\mathrm{pHs}$, resulting in faster or slower transformation.

$\mathrm{OCT}$ imaging was explored with stimuli-responsive $\mathrm{Au}$ NCs to detect distinct signal changes including scattering intensity and Doppler variance. Acid-disassembling $\mathrm{Au}$ NCs were incubated in DI water and $\mathrm{pH} 5.0$ at $37^{\circ} \mathrm{C}$ for $2 \mathrm{~h}$, as in Fig. 4. Then Au NC-containing droplets $(3 \mu \mathrm{L})$ were imaged using spectral domain (SD)-OCT and DvOCT at the same focal points (Fig. 4). The high SPR effect associated with closely packed plasmonic Au NPs 

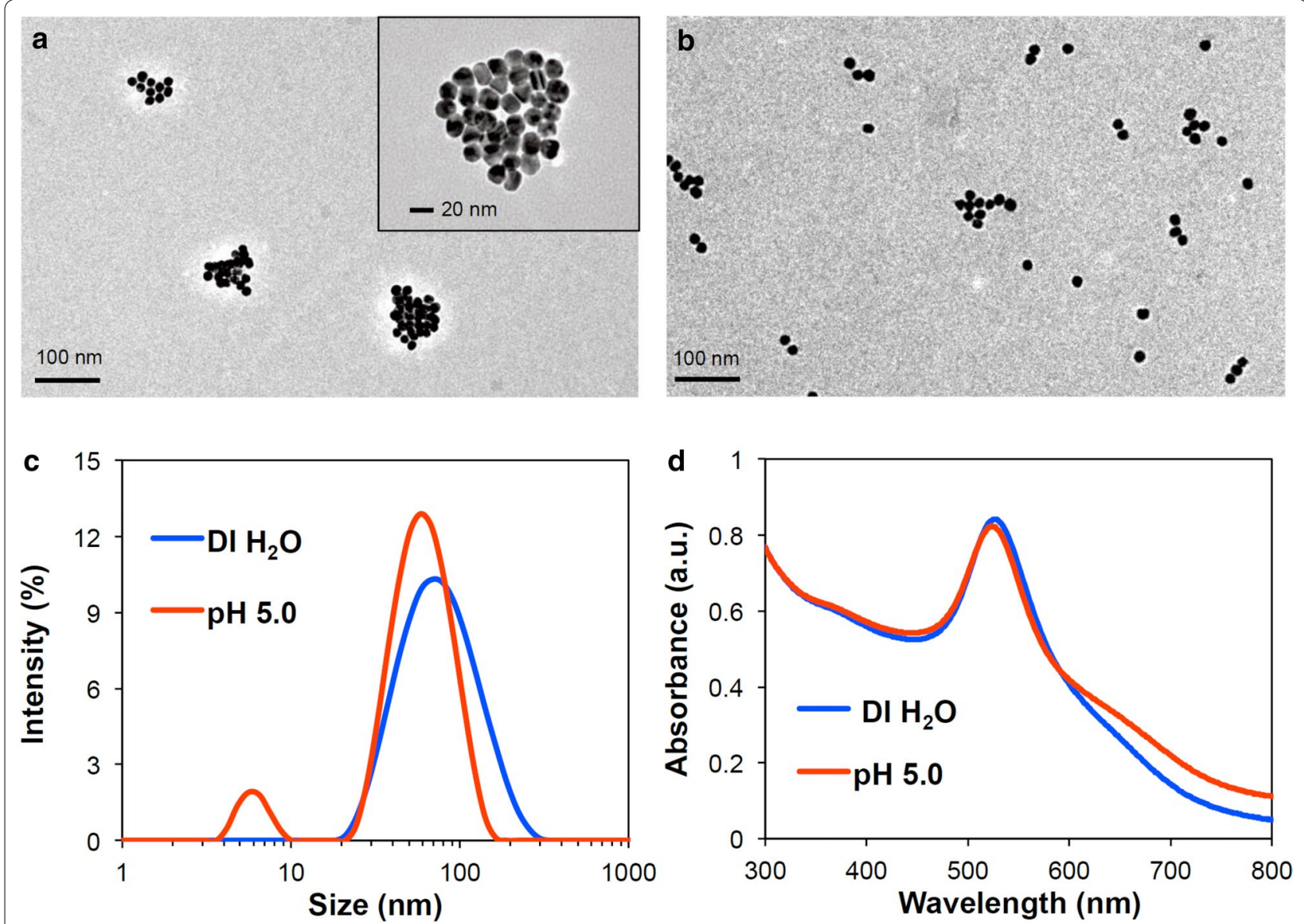

Fig. 3 Characterization of Au NCs. Acid-responsive changes in size and morphology of Au NCs incubated in DI water and 100 mM acetate buffer (pH 5.0) at $37^{\circ} \mathrm{C}$ for $2 \mathrm{~h}$, confirmed by TEM (a DI water; b pH 5.0), DLS (c), and UV-Vis spectra (d)

caused acid-disassembling Au NCs in DI water (Fig. 4a) to generate substantially stronger scattering signals than the sample in pH 5.0 buffer (Fig. 4b). For DvOCT, acid-disassembling Au NCs in pH 5.0 buffer (Fig. 4d) showed greater Doppler variance signal than the sample in DI water (Fig. 4c; the appearance of fast moving particles [colored red]) upon disassembly of relatively large $\mathrm{Au}$ NCs to smaller, more mobile Au NPs. Disassembled individual Au NPs $(15 \mathrm{~nm})$ have the absorption dominant optical property, compared to assembled Au NPs $(85 \mathrm{~nm})$. Doppler variance of all pixels in DvOCT images was quantified. The quantitative plot (Fig. 4e) showed an obvious increase in Brownian motion brought about by $\mathrm{Au}$ NC disassembly at pH 5.0 as compared to in DI water. Taken together, proof-of-concept experiments (Figs. 3, 4) clearly demonstrated acid-triggered disassembly of $\mathrm{Au}$ NCs generates distinct optical signal changes in OCT and DvOCT images.

\subsection{Confirmation of mildly acidic pH in early-stage oral dysplasia}

Low $\mathrm{pH}$ condition, especially, $\mathrm{pH}$ 5.0, is a commonly employed stimulus in acid-responsive intracellular drug delivery as endosome-mimicking condition [45]. However, real extracellular $\mathrm{pH}$ in tumor tissue contributed to by many different molecular and physiological changes during carcinogenesis $[46,47]$ greatly varies in the range of $\mathrm{pH}$ 6.5-7.2. Therefore, it was necessary to confirm the $\mathrm{pH}$ of early oral dysplasia. In this study, a hamster cheek pouch model was used as an early-stage oral dysplasia model because it has a variety of common features with human oral cancer including neovascularization during carcinogenesis, oncogenic expression, and immune response [48]. A proven carcinogen (9,10-dimethyl1,2-benzanthracene (DMBA)) was used to induce squamous neoplasia. To measure the $\mathrm{pH}$ of the tissue from the animal model, a pH-sensitive fluorescent dye, carboxyl 


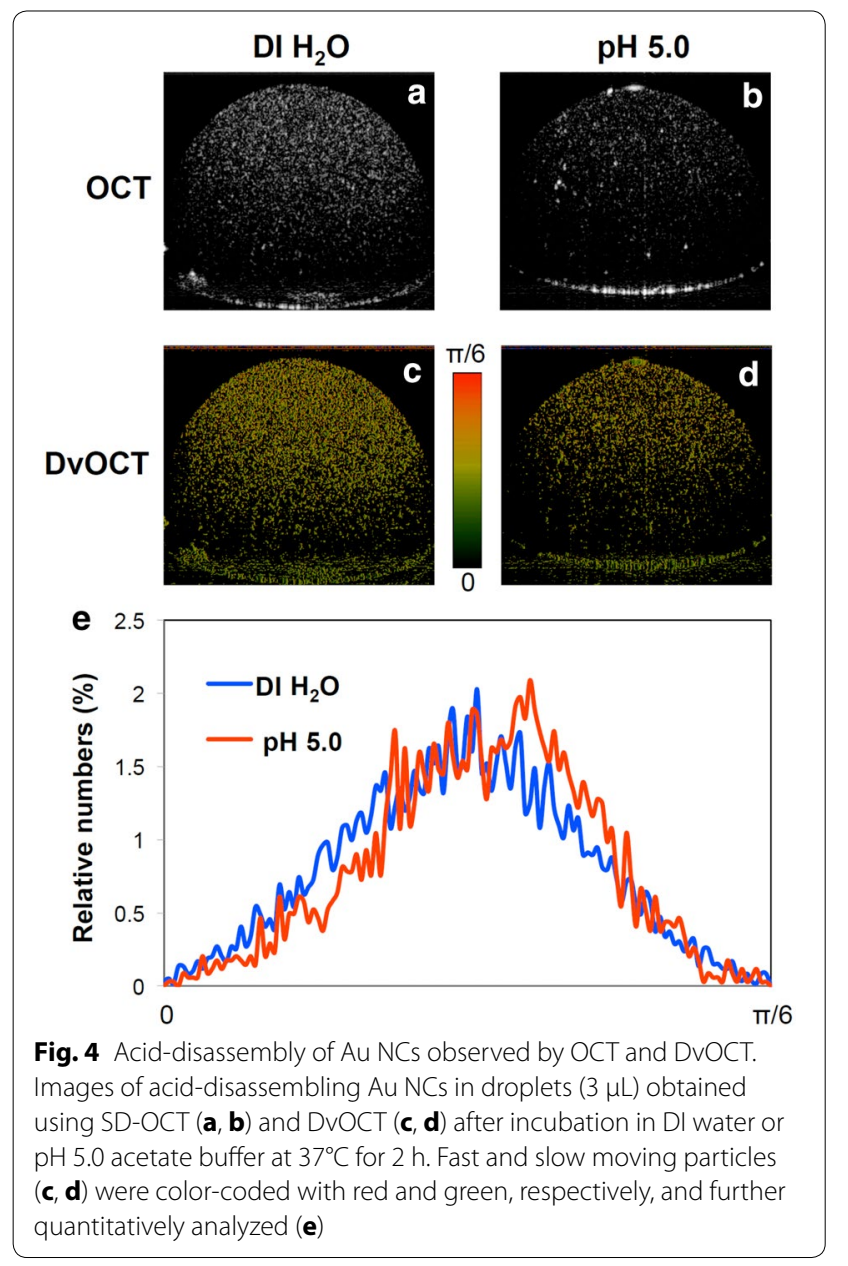

SNARF, was conjugated (Au NC-SNARF) to the Au NC surface following the general method described in Fig. 1. SNARF on Au NC surface generates $\mathrm{pH}$-dependent fluorescent emission ratios at $580 \mathrm{~nm}$ to 640 wavelengths upon excitation by a $488 \mathrm{~nm}$ wavelength light [49]. Before beginning animal experiments, the fluorescence of $\mathrm{Au}$ NC-SNARF at $580 \mathrm{~nm}$ vs. $640 \mathrm{~nm}$ emission wavelengths in varying $\mathrm{pH}$ 5.0-7.5 buffers was compared to obtain a standard curve (Fig. 5a). Then Au NC-SNARFs were topically administered on the DMBA-treated and control (mineral oil-treated) hamster cheek pouches using the combined microneedle and ultrasound delivery method as was reported previously [27]. Confocal images of the DMBA-treated cheek pouch and control cheek pouch were taken at $580 \mathrm{~nm}$ and $640 \mathrm{~nm}$ emission wavelengths with excitation at $488 \mathrm{~nm}$ wavelength. Then images in 50 by 50 pixels were obtained and processed to quantify fluorescence intensities at $580 \mathrm{~nm}$ vs. $640 \mathrm{~nm}$. As shown in Fig. $5 \mathrm{~b}$ and Additional file 1: Figure S1, the $\mathrm{pH}$ in the DMBA-applied cheek pouch tissue was determined to be mildly acidic (approximately 6.2), while the $\mathrm{pH}$ in the mineral oil-applied cheek pouch tissue was found to be close to a normal physiological condition (approximately 7.3).

After the fluorescence imaging in vivo, the oral tissues were collected and stained with hematoxylin and eosin (H\&E) to histopathologically confirm early-stage oral dysplasia in the DMBA-applied cheek pouch compared to normal tissue (Additional file 1: Figures S2 and Fig. 6). The image of the DMBA-applied tissue showed thickened
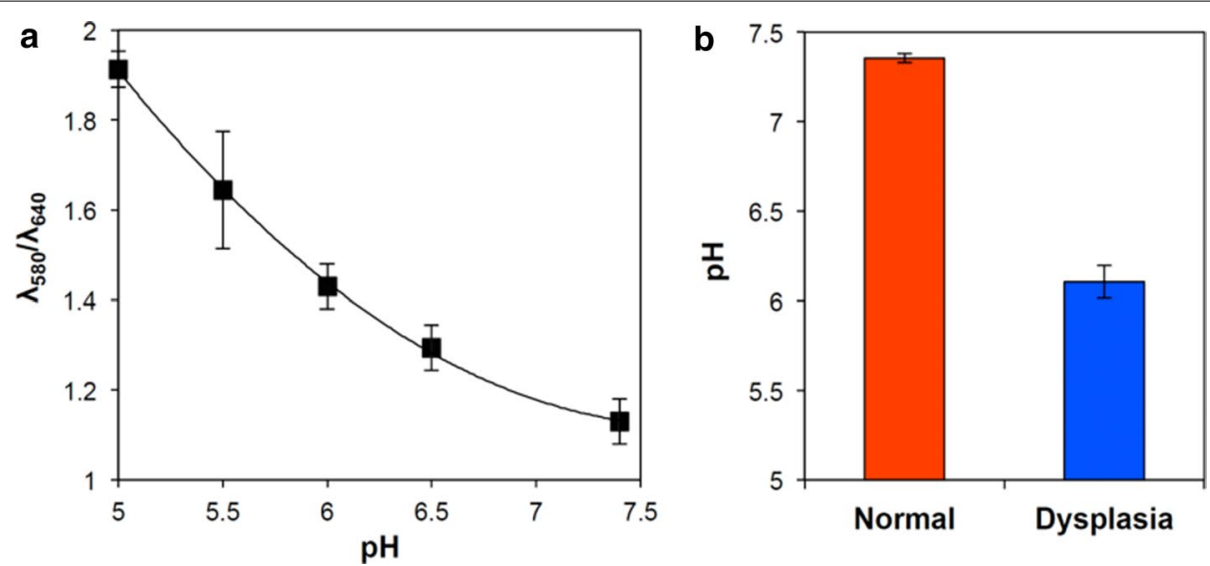

Fig. 5 Characterization of $\mathrm{pH}$ in normal and dyplastic tissue. pH-dependent fluorescence ratios of Au NC-SNARF at 580 and 640 nm wavelengths $\left(\lambda_{580 \mathrm{~nm}} / \lambda_{640 \mathrm{~nm}}\right)$ at various pHs (standard curve) (a) and pHs in normal (applied with mineral oil alone) and oral dysplastic (applied with DMBA in mineral oil) tissues, determined by topically applying Au NC-SNARF using multi-modal delivery methods and comparing resultant fluorescence ratios by confocal microscopy at 580-640 nm wavelengths in the tissues using confocal microscopy (b) 
epithelial layers and early-stage epithelial down growth underneath of the stratum cornea (circled area in Fig. 6a), indicating an early-stage dysplastic event, while the epithelial layers remained unchanged in the contralateral cheek pouch (Fig. 6b).

In addition, no adverse effects (e.g., inflammation) from the $\mathrm{Au}$ NCs were observed in either cheek pouch tissue sample, implying high biocompatibility of the administration methods. The results shown in Figs. 5 and 6 confirm that mild acidification ( $\mathrm{pH}$ 6.2) is indeed a promising pathological trigger in early-stage oral dysplasia for $\mathrm{Au}$ NC disassembly. We previously reported that the ketal linkage used in synthesizing Au NCs in this study readily hydrolyzes not only at $\mathrm{pH} 5.0$ but also at pH 6.0 in cell culture [43]. Therefore, acid-triggered Au NC disassembly and simultaneous generation of optical signal changes are anticipated in early-stage oral dysplasia ( $\mathrm{pH}$ 6.2).

\subsection{Pinpointed, multi-modal, optical diagnosis of early-stage oral cancer using acid-disassembling $\mathrm{Au}$ $\mathrm{NCs}$}

Acid-disassembling $\mathrm{Au}$ NCs were topically administered on the DMBA-applied hamster cheek pouch (oral dysplasia) and the other control cheek pouch (normal) as described earlier. In order to minimize OCT signal changes resulting from measurement errors such as tissue movement and different scattering coefficient of skin at different imaging depth, [50] a constant focal point of imaging in the cheek pouch of the anesthetized hamsters was maintained. Both scattering and Doppler variance images were obtained using SD-OCT and DvOCT, respectively, at different time points after $\mathrm{Au} \mathrm{NC}$ administration (Fig. 7).

First of all, very minimal scattering optical signals were obtained before administering $\mathrm{Au}$ NCs (base in Figs. 7a, 8). The Doppler variance in epithelial layers in the oral dysplasia tissue without $\mathrm{Au} \mathrm{NCs}$ (base) was lower than that in normal tissue (Fig. 7b, c), possibly due to the tight junctions between the overgrown epithelial cells (Fig. 6a). However, when acid-disassembling $\mathrm{Au}$ NCs were administered, significantly improved scattering images (Figs. 7a and 8) and Doppler variance images (Fig. 7b) were obtained from dysplastic tissue and from normal tissue (Additional file 1: Figures S3 and S4). OCT images clearly showed irregular stratification of the epithelial layers beneath the stratum cornea (bright top line) in oral dysplasia (Fig. 7a), while normal stratification of the epithelial layers was found in control tissue (Additional file 1: Figures S3 and S4). As hypothesized, lower scattering intensity was obtained in the oral dysplastic tissue than in normal tissue (Figs. 7, 8). Administration of acid-disassembling Au NCs also significantly improved DvOCT images (Additional file 1: Figures S3; Figs. 7b, and 8). Most notably, the Doppler variance in the epithelial layers (yellow area) substantially increased in the dysplasia (increased red pixels as compared to the base case in Fig. 7b). The epithelial layers are the sites of mild acidity as caused by localized early-stage dysplasia (Figs. 5, 6). Also, Doppler variance became even higher in the dysplastic tissue than that in the normal tissue (Additional file 1: Figures S3 and Fig. 7c). Therefore, the results shown in Fig. 7 demonstrated acid-triggered disassembly of Au NCs to Au NPs by increased Doppler variance (Brownian motion) in the epithelial layers in the dysplastic tissue.

In contrast, the Doppler variance in epithelial layers in the dysplastic tissue was lower than that in the normal tissue, when non-acid-disassembling Au NCs (control $\mathrm{Au} \mathrm{NCs}$ ) were administered (Fig. 9c). In general, contrast of OCT and DvOCT images in the dysplastic tissue were enhanced by applying non-acid-degradable $\mathrm{Au}$ NCs (Figs. 8, 9a, b), indicating stimuli-independent optical contrast enhancement by both types of Au NCs. However, the scattering signal changes in the dysplastic tissue treated with non-acid-disassembling Au NCs, in comparison with normal tissue (Additional file 1: Figures S3 and S4), was smaller than when acid-disassembling $\mathrm{Au}$ NCs were administered. Moreover, higher Doppler
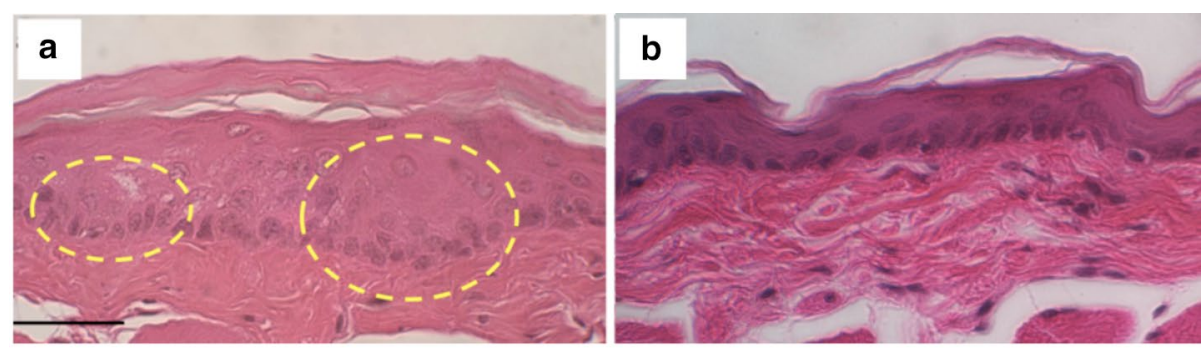

Fig. 6 H\&E stained dysplastic hamster cheek pouch tissue topically administered with acid-disassembling Au NCs (a) and normal hamster cheek pouch tissue $(\mathbf{b})$. Dotted circles indicate thickened epithelial layers indicating early-stage dysplasia. Scale bar: $100 \mu \mathrm{m}$ 

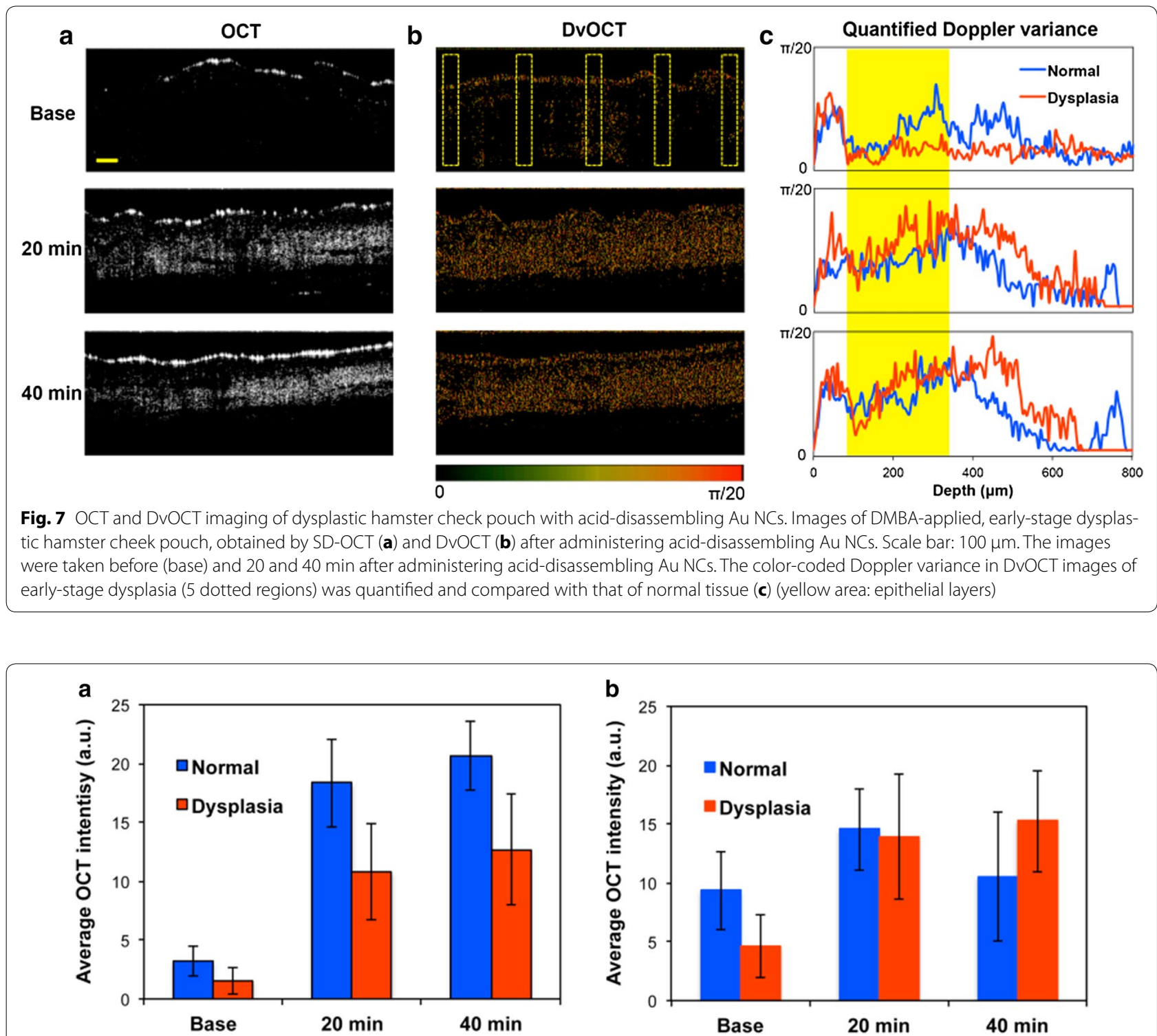

Fig. 8 Quantification of OCT results. Average SD-OCT intensity for a acid-degradable Au-NCs and $\mathbf{b}$ non-acid degradable Au-NCs after before (base) and after multi-modal delivery method (20 and 40 min) in 5 different dotted SD-OCT image regions in Figs. 7 and Additional file 1: Figure S3

variance in the epithelial layers in the dysplastic tissue compared with the normal tissue was found only when acid-disassembling $\mathrm{Au}$ NCs were administered (Figs. 7, 9). Therefore, the results shown in Figs. 7 and 9 clearly demonstrate the detection of early-stage dysplasia with pinpoint accuracy, which is challenging to confirm with most imaging modalities, by obtaining stimuli-triggered, multimodal (scattering and Doppler variance) optical signal changes using novel nanoparticle-based optical contrast agents.

\section{Conclusions}

This study demonstrates the high feasibility of identifying neoplastic change by employing stimuli-responsive contrast agents for a clinically relevant optical imaging system (i.e., optical coherence tomography). A clustered form of Au NPs (i.e., Au NCs) exhibited enhanced scattering and slow Brownian motion in normal tissue, while Au NCs reverted to individual Au NPs and showed diminished scattering and fast Brownian motion upon acid-hydrolysis of the clustering linkers in mildly acidic 


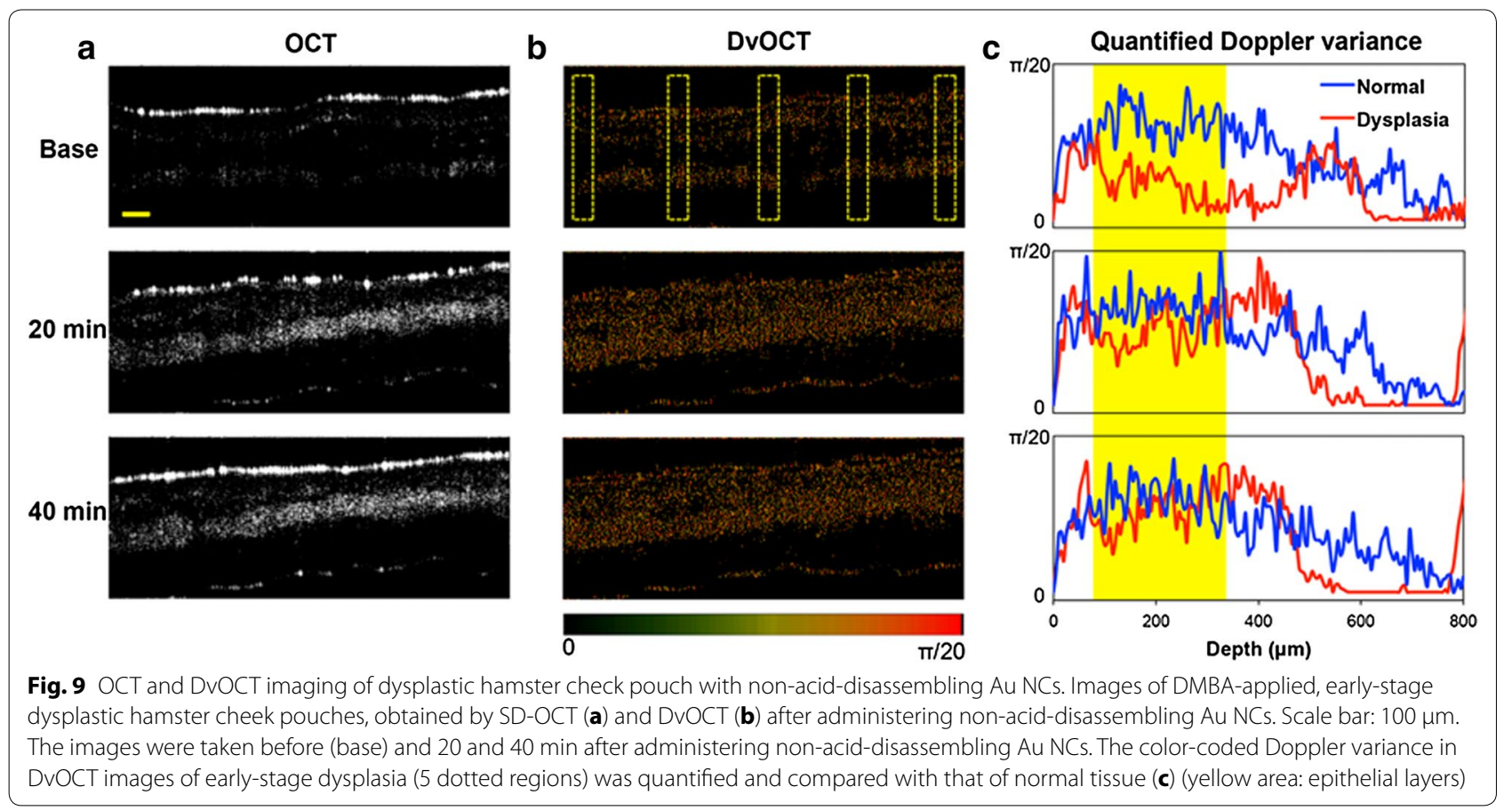

early-stage dysplastic tissue. This study's hypothesis of obtaining multiple, stimuli-responsive optical signal changes using molecularly engineered inorganic NPs can further be expanded to drug delivery and other imaging applications. Stimuli-transforming clusters can be synthesized using a variety of nanomaterials and tuned to be responsive to specific pathological stimuli (e.g., hyperthermia, enzymes, and hypoxia) as well as external triggers (e.g., magnetic force, electric field, and light). Combined incorporation of therapeutic and diagnostic (imaging) nanomaterials can be used to develop novel theranostic agents.

\section{Additional file}

Additional file 1. Additional figures and tables.

\section{Authors' contributions}

CSK planned and executed this study and wrote the manuscript. DI fabricated nanoclusters, completed in vitro studies, and assisted in writing the manuscript. PW and ZC served as expert consultants throughout the duration of the study. YJK directed the study, designed experiments, and wrote the manuscript. All authors read and approved the final manuscript.

\section{Author details}

${ }^{1}$ Department of Chemical Engineering and Materials Science, University of California, Irvine, 916 Engineering Tower, Irvine, CA 92697-2575, USA. ${ }^{2}$ University of California, Irvine, Beckman Laser Institute, 1002 Health Sciences Road East, Irvine, CA 92612, USA. ${ }^{3}$ Department of Biomedical Engineering, University of California, Irvine, 3120 Natural Sciences II, Irvine, CA 92697-2715, USA. ${ }^{4}$ Department of Pharmaceutical Sciences, University of California, Irvine,
147 Bison Modular, Irvine, CA 92697, USA. ${ }^{5}$ Department of Molecular Biology and Biochemistry, University of California, Irvine, 3205 McGaugh Hall, Irvine, CA 92697-3900, USA. ${ }^{6}$ Department of Chemical Engineering and Materials Science, University of California, Irvine, 1002 Health Sciences Rd, Irvine, CA 92617, USA. ${ }^{7}$ Department of Pharmaceutical Sciences, University of California, Irvine, 132 Sprague Hall, Irvine, CA 92697, USA.

\section{Acknowledgements}

The authors thank undergraduate researchers (Travis Tucker, Joseph Youssef, and Steven Duong) in Dr. Petra Wilder-Smith's group for their assistance in animal handling for in vivo studies. Also, the authors acknowledge Dr. Tatiana Krasieva for confocal imaging, Laurie Newman for animal care, and Hongrui Li for histology of tissue samples, at the Beckman Laser Institute at UC Irvine. DI was support by an NSF GRFP Fellowship. ZC has a financial interest in OCT Medical, Inc., which, however, did not support this work.

\section{Competing interests}

The authors declare that they have no competing interests.

Availability of data and materials

The authors have no data to share since all data are shown in the submitted manuscript.

\section{Funding}

This work was financially supported by the NIH (3R21DE19298-02S1, R01EB10090, P41EB-015890), AFOSR (FA9550-04-0101), the Beckman Laser Institute Endowment, the UC Cancer Center Support Grant (5P30CA062203-13), UC CRCC 53082, and a Council on Research Computing and Libraries award (UCI).

\section{Publisher's Note}

Springer Nature remains neutral with regard to jurisdictional claims in published maps and institutional affiliations.

Received: 5 December 2017 Accepted: 11 January 2018

Published online: 26 January 2018 


\section{References}

1. P.K. Jain, X. Huang, I.H. El-Sayed, M.A. El-Sayed, Plasmonics 2, 107 (2007)

2. H. Wang, D.W. Brandl, F. Le, P. Nordlander, N.J. Halas, Nano Lett. 6, 827 (2006)

3. M.A. Dobrovolskaia, S.E. McNeil, Nat. Nanotechnol. 2, 469 (2007)

4. N.G. Portney, M. Ozkan, Anal. Bioanal. Chem. 384, 620 (2006)

5. Z. Chen, H. Meng, G. Xing, C. Chen, Y. Zhao, Int. J. Nanotechnol. 4, 179 (2007)

6. C.S. Kim, G.Y. Tonga, D. Solfiell, V.M. Rotello, Adv. Drug Deliv. Rev. 65, 93 (2013)

7. A.M. Gobin, M.H. Lee, N.J. Halas, W.D. James, R.A. Drezek, J.L. West, Nano Lett. 7, 1929 (2007)

8. X. Huang, P.K. Jain, I.H. El-Sayed, M.A. El-Sayed, Lasers Med. Sci. 23, 217 (2008)

9. M. Everts, V. Saini, J.L. Leddon, R.J. Kok, M. Stoff-Khalili, M.A. Preuss, C.L. Millican, G. Perkins, J.M. Brown, H. Bagaria, D.E. Nikles, D.T. Johnson, V.P. Zharov, D.T. Curiel, Nano Lett. 6, 587 (2006)

10. H. Jin, K.A. Kang, Application of novel metal nanoparticles as optical/thermal agents in optical mammography and hyperthermic treatment for breast cancer. Oxygen transport to tissue (Springer, New York, 2008), pp. 45-52

11. X. Huang, P.K. Jain, I.H. El-Sayed, M.A. El-Sayed, Photochem. Photobiol. 82, $412(2006)$

12. D.P. O'Neal, L.R. Hirsch, N.J. Halas, J.D. Payne, J.L. West, Cancer Lett. 209, $171(2004)$

13. Z. Ding, H. Ren, Y. Zhao, J.S. Nelson, Z. Chen, Opt. Lett. 27, 243 (2002)

14. W. Drexler, J. Biomed. Opt. 9, 47 (2004)

15. D. Huang, E.A. Swanson, C.P. Lin, J.S. Schuman, W.G. Stinson, W. Chang, M.R. Hee, T. Flotte, K. Gregory, C.A. Puliafito et al., Science 254, 1178 (1991)

16. W. Jung, J. Zhang, J. Chung, P. Wilder-Smith, M. Brenner, J.S. Nelson, Z. Chen, IEEE J. Sel. Top. Quantum Electron. 11, 811 (2005)

17. S.A. Boppart, W. Luo, D.L. Marks, K.W. Singletary, Breast Cancer Res. Treat. $\mathbf{8 4}, 85$ (2004)

18. J. Welzel, E. Lankenau, R. Birngruber, R. Engelhardt, J. Am. Acad. Dermatol. 37, 958 (1997)

19. S.A. Boppart, A.L. Oldenburg, C. Xu, D.L. Marks, J. Biomed. Opt. 10, 41208 (2005)

20. M.J. Cobb, Y. Chen, S.L. Bailey, C.J. Kemp, X. Li, Cancer Biomarkers 2, 163 (2006)

21. P. Wilder-Smith, W.-G. Jung, M. Brenner, K. Osann, H. Beydoun, D. Messadi, Z. Chen, Lasers Surg. Med. 35, 269 (2004)

22. C. Jesser, S. Boppart, C. Pitris, D. Stamper, G.P. Nielsen, M. Brezinski, J. Fujimoto, Br. J. Radiol. 72, 1170 (1999)

23. K. Sokolov, M. Follen, J. Aaron, I. Pavlova, A. Malpica, R. Lotan, R. RichardsKortum, Can. Res. 63, 1999 (2003)

24. A.L. Oldenburg, W. Luo, S.A. Boppart, Boppart Biomed. Optics 6097, 609702 (2006)

25. T.M. Lee, A.L. Oldenburg, S. Sitafalwalla, D.L. Marks, W. Luo, F.J. Toublan, K.S. Suslick, S.A. Boppart, Opt. Lett. 28, 1546 (2003)

26. J.C. Kah, M. Olivo, T.H. Chow, K. San Song, K.Z. Koh, S. Mhaisalkar, C.J. Sheppard, J. Biomed. Optics 14, 054015 (2009)
27. C.S. Kim, P. Wilder-Smith, Y.-C. Ahn, L.-H.L. Liaw, Z. Chen, Y.J. Kwon, J. Biomed. Optics 14, 034008 (2009)

28. K. Engin, D. Leeper, J. Cater, A. Thistlethwaite, L. Tupchong, J. McFarlane, Int. J. Hyperth. 11, 211 (1995)

29. Z. Cheng, A Al Zaki. J Z Hui, V R Muzykantov and A Tsourkas, Science 338 $903(2012)$

30. V. Estrella, T. Chen, M. Lloyd, J. Wojtkowiak, H.H. Cornnell, A. IbrahimHashim, K. Bailey, Y. Balagurunathan, J.M. Rothberg, B.F. Sloane, Can. Res. 73, 1524 (2013)

31. J.M. Liu, J.T. Chen, X.P. Yan, Anal. Chem. 85, 3238 (2013)

32. J. Peng, T. Qi, J. Liao, B. Chu, Q. Yang, Y. Qu, W. Li, H. Li, F. Luo, Z. Qian, Theranostics 4, 678 (2014)

33. D. Li, Q. He, Y. Cui, J. Li, Chem. Mater. 19, 412 (2007)

34. R. Sardar, N.S. Bjorge, J.S. Shumaker-Parry, Macromolecules 41, 4347 (2008)

35. C.S. Kim, W. Qi, J. Zhang, Y.J. Kwon, Z. Chen, Journal of biomedical optics 18, 030504 (2013)

36. H. Ren, K.M. Brecke, Z. Ding, Y. Zhao, J.S. Nelson, Z. Chen, Opt. Lett. 27, 409 (2002)

37. G. Frens, Nature 241, 20 (1973)

38. W. Haiss, N.T. Thanh, J. Aveyard, D.G. Fernig, Anal. Chem. 79, 4215 (2007)

39. J.P. Novak, C. Nickerson, S. Franzen, D.L. Feldheim, Anal. Chem. 73, 5758 (2001)

40. P. Wilder-Smith, M.J. Hammer-Wilson, J. Zhang, Q. Wang, K. Osann, Z Chen, H. Wigdor, J. Schwartz, J. Epstein, Clin. Cancer Res. 13, 2449 (2007)

41. A. Oldenburg, D.A. Zweifel, C. Xu, A. Wei, S.A. Boppart, Biomed. Optics 5703, 50 (2005)

42. J.A. MacKay, M. Chen, J.R. McDaniel, W. Liu, A.J. Simnick, A. Chilkoti, Nat. Mater 8993 (2009)

43. M.S. Shim, Y.J. Kwon, Biomacromol 9, 444 (2008)

44. S.D. Perrault, C. Walkey, T. Jennings, H.C. Fischer, W.C. Chan, Nano Lett. 9 1909 (2009)

45. D. Schmaljohann, Adv. Drug Deliv. Rev. 58, 1655 (2006)

46. S. Mura, J. Nicolas, P. Couvreur, Nat. Mater. 12, 991 (2013)

47. J.A. Menendez, R. Lupu, Nat. Rev. Cancer 7, 763 (2007)

48. B. Mognetti, F. Di Carlo, G. Berta, Oral Oncol. 42, 448 (2006)

49. R.C. Hunter, T.J. Beveridge, Appl. Environ. Microbiol. 71, 2501 (2005)

50. R.O. Esenaliev, K.V. Larin, I.V. Larina, M. Motamedi, Opt. Lett. 26, 992 (2001)

\section{Submit your manuscript to a SpringerOpen ${ }^{\circ}$ journal and benefit from:}

- Convenient online submission

- Rigorous peer review

- Open access: articles freely available online

- High visibility within the field

- Retaining the copyright to your article

Submit your next manuscript at springeropen.com 